\title{
Magnetic Actuator for a Capsule Endoscope Navigation System
}

\author{
Atsushi Chiba, Masahiko Sendoh, Kazushi Ishiyama*, Ken Ichi Arai, \\ Hironao Kawano ${ }^{1}$, Akio Uchiyama ${ }^{1}$, and Hironobu Takizawa ${ }^{1}$ \\ Research Institute of Electrical Communication, Tohoku University, 2-1-1 Katahira Aoba Sendai 980-8577, Japan \\ ${ }^{1}$ Olympus Medical Systems Corp., 2951 Ishikawa-cho Hachioji Tokyo 192-8507, Japan
}

(Received 24 December 2005)

\begin{abstract}
The authors propose a magnetic actuator for use as a navigation system for capsule endoscopes. The actuator is composed of a capsule dummy, a permanent magnet inside the capsule, and an external spiral structure. The device rotates and propels wirelessly when exposed to an external rotational magnetic field. In this study we measured the effect of the spiral shape on the velocity and thrust force properties. According to our experimental results, the actuator obtained a maximum velocity and thrust force when the spiral angle was set at 45 degrees, the number of spirals was set at 4 , and the spiral-height was set at 1-mmf. We also conducted a motion test in the large intestine of a pig placed on a 30 degrees slope. The actuator passed through a $700 \mathrm{~mm}$ length of the intestine in about $300 \mathrm{~s}$. The device also managed to travel up and down the 30 degrees slope with no difficulty whatsoever. Our results demonstrate the great potential of this actuator for use as a navigation system for capsule endoscopes.
\end{abstract}

Keywords : magnetic actuator, magnetic torque, spiral structure

\section{Introduction}

Gastrointestinal (GI) endoscopy is a widely used procedure to find diseases in the early stages. A capsule endoscope is a pill-sized inspection device that can be easily swallowed [1,2]. The small size of the device and simplicity of the procedure minimize the suffering of the patient. Lacking any internal mechanisms for locomotion, the capsule moves passively through the GI tract with the aid of peristalsis. This is an important shortcoming, as it precludes external control of the device trajectory and increases the chance that disease sites will be bypassed unnoticed. An active, self-driving mechanism is therefore desired. Several locomotion devices have been proposed for application in the gastrointestinal lumen [3-5]. None can be used for movable devices such as capsule endoscopes, however, as none can be driven wirelessly.

In our previous study we introduced a magnetic actuator with an internal magnet and an external spiral structure that can be driven wirelessly when exposed to an external rotational field [6-8]. The direction of travel of the magnetic actuator can be controlled by changing the

*Corresponding author: Tel: +81-22-217-5488,

Fax: +81-22-217-5728, e-mail: ishiyama@riec.tohoku.ac.jp rotational plane of the field. A separate report has described the effects of the spiral structure on the movement of a capsule-type magnetic actuator [9]. This type of actuator has also been confirmed to have the ability to pass through an intestine extracted from a pig [10, 11]. In this study we measured the effect of the spiral structure on the velocity and thrust force properties of this magnetic actuator. We also performed a moving test in a pig intestine oriented on a slope.

\section{Capsule-type Magnetic Actuator}

Fig. 1 shows a conceptual illustration of a capsule-type magnetic actuator. The device is composed of a permanent magnet inside the capsule and a spiral structure outside. The permanent magnet is magnetized in the direction of the diameter. When a rotational magnetic field is applied, the magnetic torque rotates the actuator, inducing locomotion wirelessly. We fabricated a capsule-type magnetic actuator using a resinoid dummy capsule. Fig. 2 shows a schematic view of the device. The capsule measures 11 $\mathrm{mm}$ in diameter and $40 \mathrm{~mm}$ in length. An $\mathrm{NdFeB}$ magnet magnetized in the direction of the capsule diameter is inserted into the capsule. The photographs in Fig. 3 show parts of several actuators fabricated with different spiral 


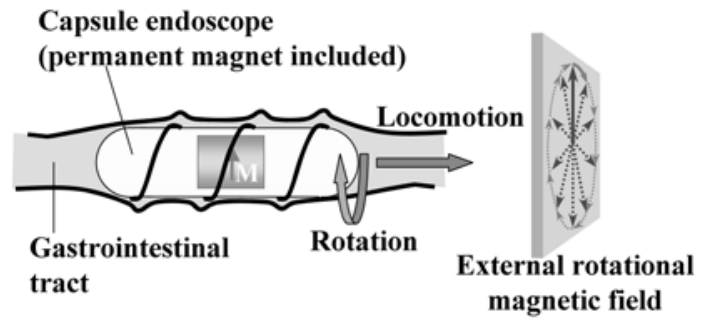

Fig. 1. Conceptual illustration of capsule endoscope utilized magnetic actuator.

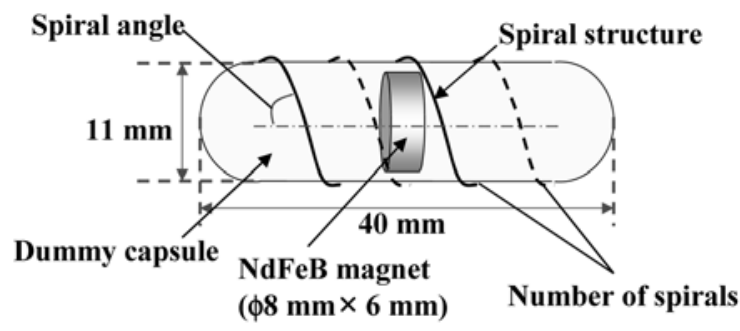

Fig. 2. Schematic view of capsule-type magnetic actuator.

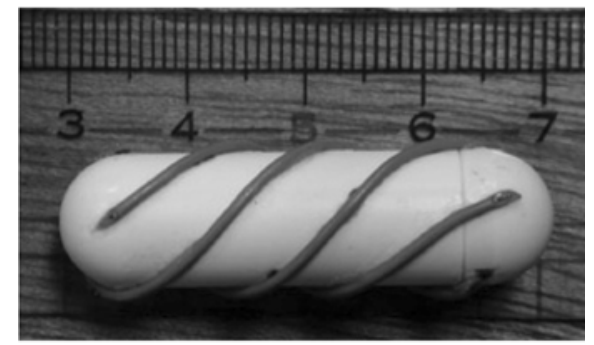

Fig. 3. Photograph of fabricated magnetic actuators (the spiral angle is 45 degree, the number of spirals is 4 , and the spiralheight is $1-\mathrm{mm} \phi)$.

structures. The spirals all have circular cross sections, but they differ in angle, number, and height. Thus, the width of the spiral structure is changed by changing the spiralheight.

\section{Basic Characteristics}

The travel velocity and thrust force of each actuator were examined using a silicone tube as a phantom. The velocity and thrust force vary in accordance with the spiral angle and number of spirals. The intensity and frequency of the rotational magnetic field applied in this experiment were $8 \mathrm{kA} / \mathrm{m}$ and $1 \mathrm{~Hz}$, respectively.

\subsection{Thrust Force}

\subsubsection{Experimental method}

The thrust force of each actuator was examined using a silicone tube as a phantom. Fig. 4 shows a schematic view of the thrust force measurement. The rotational

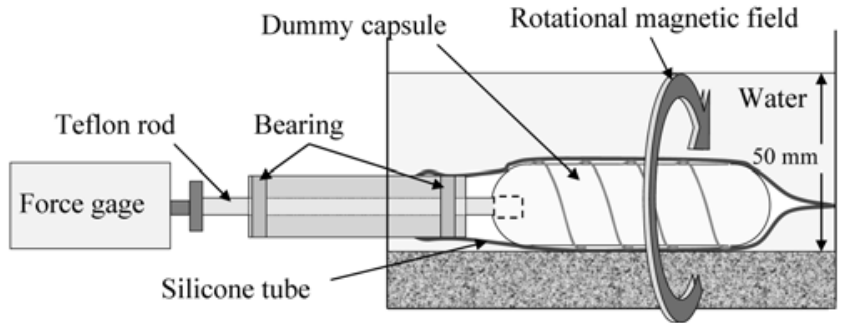

Fig. 4. Schematic view of thrust force measurement.

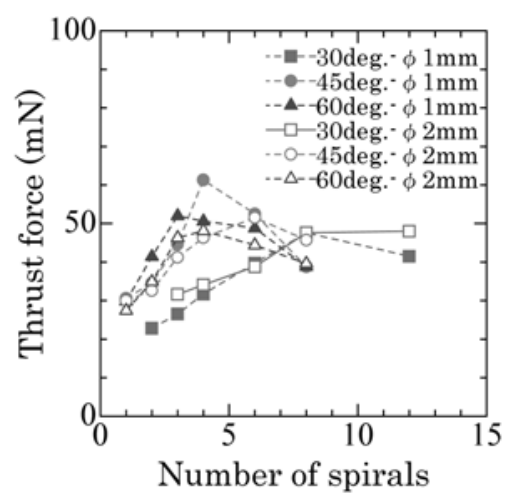

Fig. 5. Relationship between the force and number of spirals.

magnetic field was generated by a set of three pairs of coils similar in structure to three-axis Helmholtz coils, with the members of each pair set at a distance of about $200 \mathrm{~mm}$ from each other. The measurement in Fig. 4 was set in the coils. The application of the rotational magnetic field induces the actuator rotation and thrust. In this experiment we used a force gauge to measure the thrust force of each actuator.

\subsubsection{Result and discussion}

Fig. 5 shows the relationship between the thrust force and number of spirals. The figures in the graph legends are the mean spiral angle and spiral-height. The maximum thrust force was obtained by the actuator with 4 spirals measuring 1-mmf in height oriented at a spiral angle of 45 degrees. We also determined the optimal number of spirals for maximizing thrust force in each spiral structure. With too few spirals, the thrust weakened due to the insufficiency of the total spiral length. With too many spirals, the thrust weakened due to the short distances between adjacent spirals. The maximum thrust was obtained when the number, angle, and height of the spirals were optimally balanced.

\subsection{Velocity}

\subsubsection{Experimental method}

We examined the velocity of each actuator using a silicone tube as a phantom. Fig. 6 shows a schematic 


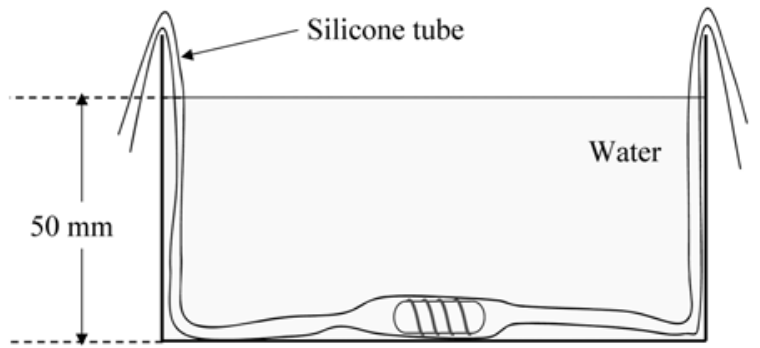

Fig. 6. Schematic view of velocity measurement.

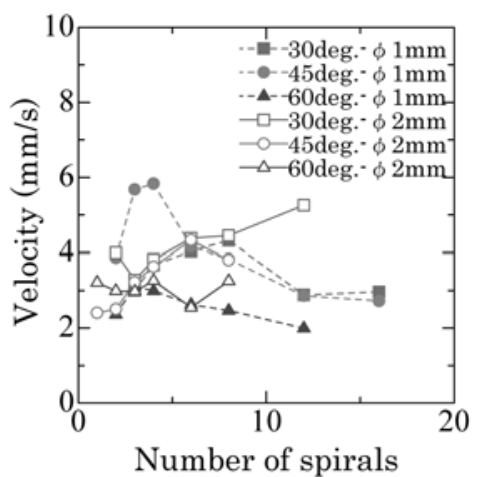

Fig. 7. Relationship between the velocity and number of spirals.

view of the velocity measurement. The velocity of each actuator was determined by measuring the traveling time and distance in the silicone tube.

\subsubsection{Result and discussion}

The relationship between the thrust force and number of spirals is shown in Fig. 7. The figures in the graph legends are the mean spiral angle and spiral height. The highest velocity was obtained in the actuator with 4 spirals measuring 1-mm $\phi$ in height and oriented at an angle of 45 degrees. As in the case with thrust force, each spiral structure had an optimal number of spirals for maximizing velocity. With too small spiral numbers, the velocity decreased due to the weaker thrust force. With too many spirals, the velocity decreased due to the short distances between adjacent spirals. In addition, the results say that there is the optimum spiral height. The reason is not clarified yet, but we are approaching to this problem by considering the difference of touching area between the actuator and the tube by changing the shape of the tube around the spiral.

\section{Moving in Intestine on Slope}

\subsection{Experimental method}

Because of the complicated structure of the human intestine, a capsule-type magnetic actuator needs the ability to propel itself both upward and downward along its

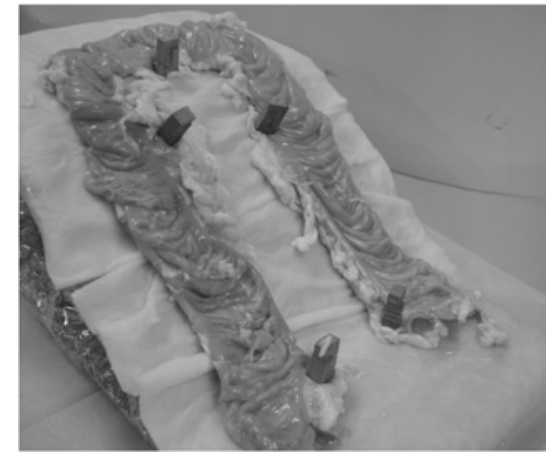

Fig. 8. Photograph of large intestine on slope.

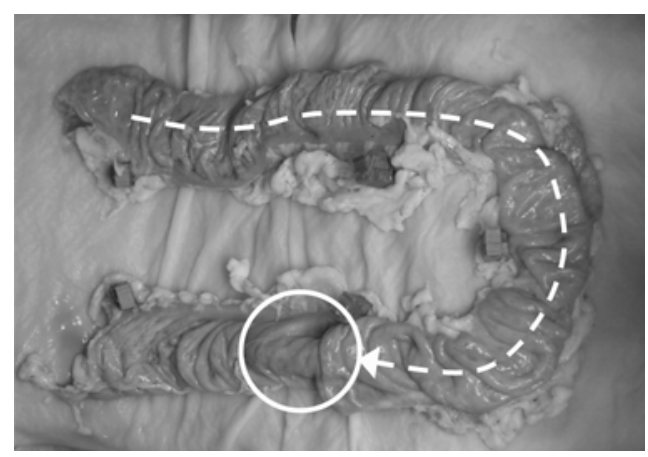

Fig. 9. Photograph of an experiment (large intestine on slope).

journey. In this experiment we performed a motion test with a capsule-type magnetic actuator in a large intestine excised from a pig. The actuator consisted of 4 spirals measuring 1-mm $\phi$ in height and oriented at 45 degrees. Fig. 8 shows a $700 \mathrm{~mm}$ length of intestine placed on a plate sloped at 30 degrees. The plate was placed directly in the center of the coil used to supply the rotational magnetic field. The intensity and frequency of the rotational magnetic field during the experiment were $8 \mathrm{kA} / \mathrm{m}$ and below $1 \mathrm{~Hz}$, respectively.

\subsection{Result and discussion}

Fig. 9 shows the result of the moving test. The circle and broken line in the figure indicate the position and trajectory of the actuator. The device passed through the large intestine from end to end in about 300 seconds, maneuvering the 30 degrees slope in both the upward and downward directions without any difficulties. Based on this result, we report that the actuator has the potential to transport a capsule endoscope within the complicated structure of the human intestine.

\section{Summary}

The moving characteristics of each actuator were examined using a silicone tube as a phantom. The maximum 
velocity and thrust force were obtained in an actuator consisting of 4 spirals measuring $1 \mathrm{~mm} \phi$ in height and oriented at an angle of 45 degrees. We also demonstrated the wireless locomotion of a capsule-type magnetic actuator through the large intestine of a pig under a rotational magnetic field. The actuator passed through a $700 \mathrm{~mm}$ length of intestine, successfully maneuvering up and down a 30 degrees slope. These results confirm the great potential of the proposed magnetic actuator as a navigation system for capsule endoscopes.

\section{References}

[1] G. Iddan, G. Meron, A. Glukhovsky, and P. Swain "Wireless capsule endoscopy", Nature 405, 417 (2000).

[2] OLYMPUS Corporation http://www.olympus.co.jp/

[3] L. Phee, D. Accoto, A. Menciassi, C. Stefanini, M. C. Carrozza, and P. Dario, "Analysis and Development of Locomotion Devices for the Gastrointestinal Tract", IEEE Trans. Biomed. Enging. 49, 613-616 (2002).

[4] H. D. Høeg, A. B. Slatkin, J. W. Burdick, and W. S. Grundfest, "Biomechanical Modeling of the Small Intestine as Required for the Design and Operation of a Robotic Endoscope", Proc. 2000 IEEE Int. Conf. on
Robotics and Automation 1599-1606 (2000).

[5] K. Ikeuchi, K. Yoshinaka, and N. Tomita, "Low invasive propulsion of medical devices by traction using mucus", Wear 209, 179-183 (1997).

[6] T. Honda, K. I. Arai, and K. Ishiyama, "Micro-swimming mechanisms propelled by external magnetic field", IEEE Trans. Magn. 32, 5085-5087 (1996).

[7] K. Ishiyama, M. Sendoh, A. Yamazaki, and K. I. Arai, "Swimming micro-machine driven by magnetic torque", Sensors and Actuators A-91, 141-144 (2001).

[8] M. Sendoh, K. Ishiyama, and K. I. Arai, "Direction and Individual Control of Magnetic Micromachine", IEEE Trans. Magn. 38, 3356-3358 (2002).

[9] M. Sendoh, K. Ishiyama, and K. I. Arai, "Fabrication of Magnetic Actuator for Use in a Capsule Endoscope", IEEE. Trans. Magn. 39, 3232-3234 (2003).

[10] A. Chiba, M. Sendoh, K. Ishiyama, and K. I. Arai, "Basic Characteristics of a Magnetic Actuator for Capsule Endoscope", Trans. Jpn. Soc. Med. Biol. Eng. 42, 313-317 (2004).

[11] A. Chiba, M. Sendoh, K. Ishiyama, and K. I. Arai, "Moving of a Magnetic Actuator for a Capsule Endoscope in the Intestine of a Pig", J. Magn. Soc. Jpn. 29, 343-346 (2005). 